\title{
Fine-needle aspiration cytology of lepromatous leprosy
}

\author{
N. SINGH, A. BHATIA, V. K. ARORA \& \\ S. N. BHATTACHARYA* \\ Cytology Section, Departments of Pathology and *Dermatology, \\ University College of Medical Sciences and Guru Tegh Bahadur \\ Hospital, Delhi 110 095, India
}

\section{Accepted for publication 12 March 1998}

\begin{abstract}
Summary A prospective study correlating cytopathology with clinical morphology and histopathology in 22 patients with lepromatous leprosy was performed. Aspirates were taken from skin lesions in all patients. Lymph node aspirates were also performed in four patients with lymphadenopathy. Fine-needle aspirates yielded sufficient cellular material with excellent preservation of morphological detail. Diagnosis and correlation with bacillary index, clinical and histopathological findings was possible in all patients. In addition, the two patterns, partial and diffuse, of lymph node involvement could be recognized. Fine-needle aspiration cytology is a simple method for the laboratory assessment of leprosy.
\end{abstract}

\section{Introduction}

The seminal observations of Marian Ridley ${ }^{1}$ on slit smears emphasized the interpretation of the cellular exudate in conventionally stained Zeihl-Neelsen (ZN) smears. In previous papers, ${ }^{2-4}$ we expanded on this concept by studying the morphological details of the cellular exudate in MGG stained fine-needle aspiration (FNA) smears and found that precise recognition of cell type was an aid in the appropriate placement of the lesions in the Ridley-Jopling (RJ) scale. ${ }^{5}$ We also observed that FNA smears, in contrast to slit skin smears, were free of confounding epidermal squamous cells and therefore better suited for evaluating cell morphology.

In this paper, we present details of new cytological findings on fine-needle aspiration in lepromatous leprosy (LL). The relationship between the cellular infiltrate and the bacillary index (BI) at this end of the spectrum and the cytomorphological correlation with clinical subtypes in 22 patients with LL, viz. macules, plaques and nodules, are defined. Additionally, definitive cytopathological identification of two patterns of lymph node involvement has been described. 


\section{Materials and methods}

Twenty-two patients clinically diagnosed with LL presenting with a variety of skin lesions ranging from macules (4), ill defined infiltrated plaques (11) and nodules (7) were studied. Six patients, including four with erythema nodosum leprosum (ENL), had received standard multibacillary multidrug therapy for periods of up to 3 months. Multiple site aspirations of skin lesions and earlobes were done in three patients. In the remainder, a single aspiration was performed. In addition, FNA of cervical (3) and axillary (1) lymph nodes was carried out.

The diagnosis and classification in the RJ scale was based on clinical examination by a dermatologist (S.N.B.) using standard criteria. ${ }^{6}$ All patients were subjected to biopsy from the same lesion which had been sampled by FNA. Biopsies were evaluated and classified according to the histological criteria established by Ridley. ${ }^{7}$ FNA smears were evaluated independently of the biopsy findings, and the results were classified using the cytological criteria laid down in our previous study. ${ }^{4}$

Smears were specifically evaluated for cellularity (high, moderate, low), morphological details of macrophages (foamy, with negative images), accompanying inflammatory cells (lymphocytes, neutrophils, eosinophils) and BI. Negative images, seen in MGG-stained smears, were cleft-like unstained spaces $^{8}$ corresponding to globi seen with modified $\mathrm{ZN}$ stain.

The procedures of FNA/cytopuncture have been described in standard textbooks of cytopathology. ${ }^{9}$ A syringe holder is commonly advocated as an aid in creating negative pressure while aspirating. However, we have dispensed with this device and employ a 'handheld' method, the negative pressure being created by lifting the piston with the thumb. The quality and quantity of material obtained were comparable to aspirations performed using a holder. A further modification of the FNA procedure was applied to skin lesions. The lesional skin was pinched, as done for the slit-smear technique, between thumb and forefinger of one hand for about $30 \mathrm{~s}$ to blanch it, while aspirating with the other. This ensured a cellular aspirate relatively uncontaminated with blood. The aspirated material was expelled onto glass slides; the flat of another slide being used to smear the material.

All smears were air-dried and stained with MGG and modified ZN stains. Cytological criteria used in the previous study ${ }^{4}$ were used to judge the adequacy of the smears and make a diagnosis.

\section{Results}

CYTOPATHOLOGY

In all 22 patients, the aspirates were adequate with a dispersed cell population. Bacillary indices were $5+$ to $6+$.

The cytological features in skin lesions in MGG-stained smears were as follows (numbers in parentheses represent numbers of patients):

\section{Macular LL (4)}

Poorly cellular smears.

Occasional macrophage with negative images.

Few lymphocytes. 
Plaques and nodular LL (14)

Cellular smears.

Abundant foamy macrophages (AFB) in a fatty background with intra- and extracellular negative images.

Few lymphocytes.

\section{Erythema nodosum leprosum (ENL) (4)}

Cellular smears.

Foamy macrophages in a background of numerous intact and fragmented neutrophils (Figure 1).

Numerous fragmented AFB present both intracellularly in macrophages and rarely, in neutrophils; and extracellularly.

\section{Lymph node aspirates (4)}

Two distinct patterns were seen:

\section{Pattern I (3)}

\section{Cellular smears.}

Single or small groups of macrophages in a background of reactive lymphoid cells. The macrophages were foamy and had negative images and intracytoplasmic 'blue' globules

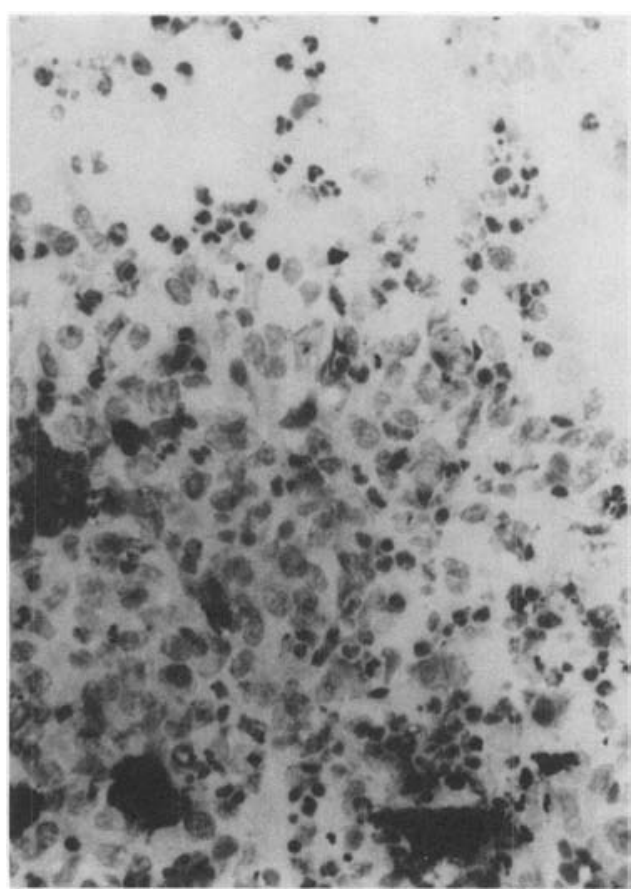

Figure 1. Erythema nodosum leprosum: neutrophils and macrophages with pale, vesicular nuclei. MCG $\times 400$. 
(Figure 2) surrounded by haloes. These globular structures corresponded to globi seen with the ZN stain.

\section{Pattern II (1)}

Highly cellular smears. The predominant, almost exclusive, cell type was macrophages, similar to those seen in pattern I. Interspersed between these were a few lymphocytes (Figure 3).

\section{HISTOPATHOLOGY}

In 15 patients the histopathology correlated well with the cytomorphology. In seven, using morphological criteria alone, the histopathological diagnosis was leprosy; however, the classification in the RJ scale was discrepant by two positions, i.e. BB-BL. These patients could correctly be reclassified as LL only on the basis of high BI in Fite-stained sections. In three instances, repeated Fite stain was required for positive results.

\section{Discussion}

In this study, all the FNA smears from macular lesions were poorly cellular, whereas plaques

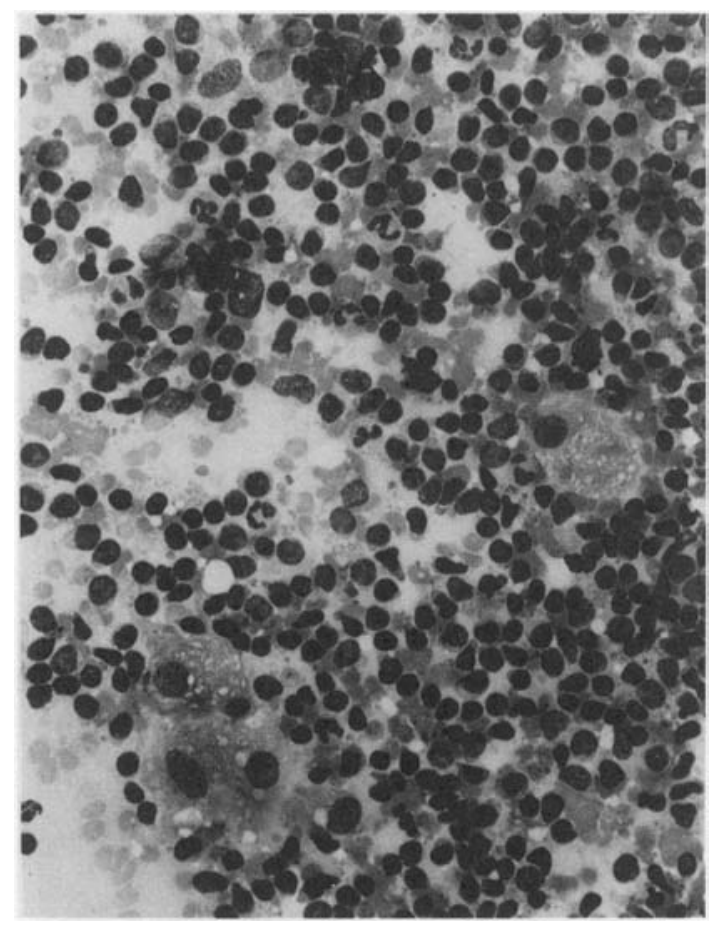

Figure 2. Partial replacement of lymph node: single, dispersed macrophages with negative images in a reactive lymphoid background. MGG $\times 100$. 


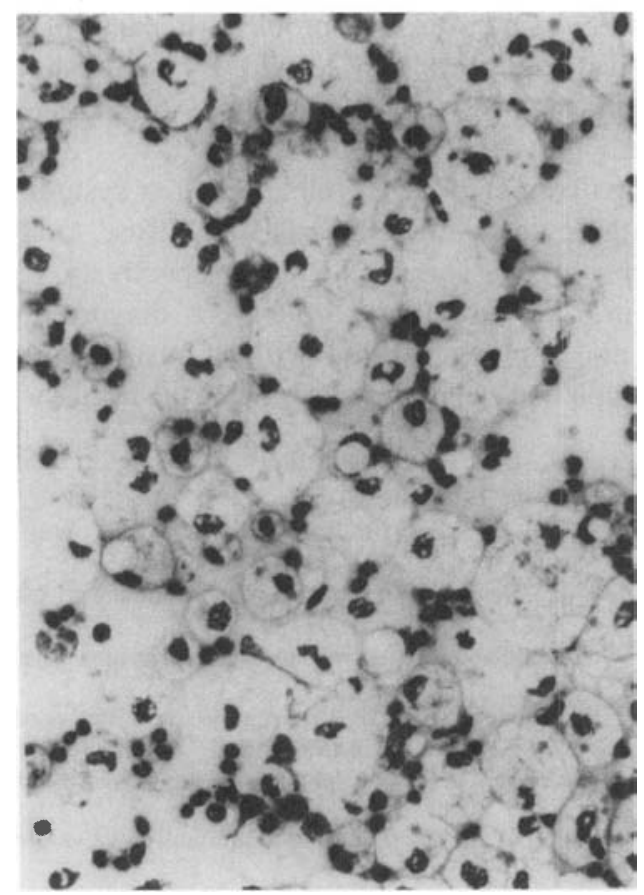

Figure 3. Complete replacement of lymph node: diffuse population of foamy macrophages. MGG $\times 100$.

and nodules had heavily cellular smears. These findings support the observation that cellularity of smears in FNA of inflammatory lesions is proportional to the amount of the infiltrate per unit volume of tissue. This, in turn, correlates with the clinical and histopathological morphology of the lesion, e.g. flat, or macular lesions have very little infiltrate, while lesions with progressively larger amounts of infiltrating cells are raised, i.e. papules, plaques or nodules.

Dispersed macrophages are seen in plaques and nodules of LL. Negative images or foamy cytoplasm correspond to high bacterial load. Mycobacteria do not stain with the Romanowsky dyes. The resulting unstained negative images have been described in other species of the genus Mycobacteriacae. ${ }^{8,10}$ Mycobacterium leprae are also visible as elongated unstained clefts in the cytoplasm which correspond to bundles of lipid rich bacteria. ${ }^{2}$ The cytological findings are sufficient independently to categorize the lesion as lepromatous. The AFB findings on the modified ZN stain correlate so well with the cell morphology on MGG stained smears that the two serve as controls to complement each other.

The presence of neutrophils, and occasionally eosinophils, with foamy macrophages points to ENL. Negative images are usually absent because the AFB are fragmented.

Architectural relationships of the inflammatory infiltrate to the various cutaneous structures are undoubtedly better appreciated in histopathological sections; however, cytologic smears are ideal for evaluation of individual cell morphology. Moreover, acid-fast bacilli are more often demonstrable in smears than on tissue section, where the stain is liable, for unexplained reasons, to fail on occasion. ${ }^{7}$ Others find biopsies better than slit-skin smears, particularly for paucibacillary leprosy. ${ }^{11}$ 
The diagnosis of LL in FNA smears is not a problem. Because of the high bacterial load in this type of disease, acid-fast bacilli are easy to find. By establishing distinct cytomorphological correlates to the clinical spectrum, FNA cytology offers a less cumbersome method for calibrating the disease.

There is generalized involvement of the lymphoreticular system, particularly the lymph nodes, in LL. Two histopathological patterns, partial and diffuse, have been described. ${ }^{12}$ This is reflected in the cytomorphology as patterns I and II, respectively. Lymph node biopsies are occasionally performed in LL, particularly when the diagnosis is missed on clinical examination, the lymph nodes being excised with a mistaken diagnosis of lymphoma. Accurate recognition of lymph node aspirates can obviate the need for biopsy in this situation.

\section{References}

${ }^{1}$ Ridley MJ. The cellular exudate-Mycobacterium leprae relationship and the critical reading of slit-smears. Lepr Rev, 1989; 60: 229-240.

2 Singh N, Arora VK, Ramam M. Nodular lepromatous leprosy: report of a case diagnosed by FNA. Diagn Cytopathol, 1994; 11: 373-375.

${ }^{3}$ Singh N, Arora VK, Bhatia A, Bhattacharya SN. A plea for routine use of fine-needle aspiration cytology in the diagnosis and followup of leprosy. Int J Lepr, 1996; 64: 84-85.

${ }^{4}$ Singh N, Bhatia A, Gupta K, Ramam M. The cytomorphology of leprosy across the Ridley-Jopling spectrum. Acta Cytol, 1996; 40: 719-723.

5 Ridley DS, Jopling WH. Classification of leprosy according to immunity. A five-group system. Int J Lepr, 1996; 34: 255-273.

6 World Health Organization. A guide to leprosy control, 2nd edn. WHO, Geneva, 1988.

7 Ridley DS. The pathogenesis of leprosy and related diseases. Butterworth \& Co, Cambridge; 1988, p 240.

${ }^{8}$ Singh N, Bhatia A, Tickoo SK, Arora VK, Gupta K. Negative-staining, refractile mycobacteria in Romanowsky stained smears. Acta Cytol, 1995; 39: 1071.

9 Orell SR, Walters MN-I, Sterrett GF, Whitaker D. Manual and atlas of fine-needle as piration cytology, Churchill Livingstone, Edinburgh; 1992, pp 8-21.

10 Tarlakovic E, Clayton F, Elizabeth DA. Refractile mycobacteria in Romanowsky stained bone marrow smears: a comparison of acid-fast stained tissue sections and Romanowsky stained smears. Am J Clin Pathol, 1992; 97: 318-321.

11 Ponnighaus JM, Lienhardt C, Lucas S, Fine PEM, Sterne JAC. Comparison of bacillary index in slit-skin smears, skin and nerve biopsies; a study from Malawi. Int J Lepr, 1997; 65: 211-216.

12 Job CK. Pathology of leprosy. In: Leprosy, 2nd edn, Hastings RC (ed.). Churchill Livingstone, London; 1994, pp 193-224. 